(Aus dem physiologischen Institut zu Breslau.)

\title{
Ueber den Gehalt des Arterien- und Venenblutes an Trockensubstanz und Fett.
}

Von

\section{F. Röhmann und J. Mühsam.}

Nachdem Flïg. $\mathrm{e}^{1}$ ) gezeigt hatte, dass die Fehler unserer Untersuchungsmethoden zu gross seien, um mit ihrer Hiulfe Veränderungen, welche das Blut beim Durchströmen von Organen, speziell der Leber erfährt, nachweisen zu können, und er ganz allgemein zu dem Resultate gekommen war, dass ,eine vergleichende Untersuchung des zu- und abführenden Blutes keine Methode sei, mittelst deren wir hoffen dürfen, einen Anfschluss iuber die Funktion der Leber zu erhalten", gehörte ein gewisser Entschluss dazu, von neuem an Versuche heranzutreten, welche bezweckten, Urtheile tiber die Funktion eines Organs durch einen Vergleich seines Arterien- und Venenblutes zu gewinnen.

Wenn es trotzdem Herr Bornstein ${ }^{2}$ ), einer Anregung von Herrn Geheimrath Heidenhain folgend, unternahm, den Fettgehalt der Carotis und Pfortader zu bestimmen, um einen Beitrag zu der Frage zu liefern, ob Fett ausser durch den Chylus auch noch direkt durch die Pfortaderwurzeln dem Organismus zugefuhrt werden, so bestimmten ihn hierzu, wie er in der Einleitung seiner Arbeit ausfuhrt, eine Reihe theils mikroskopischer Untersuchungen der Darmschleimhaut, in denen sich angeblich der Uebertritt von Fett in die Darmeapillaren hatte verfolgen lassen, theils gewisse

1) Ueber den Nachweis des Stoffwechsels in der Leber. Zeitschr. für Biologie Bd. 13, 133.

2) Einiges über die Zusammensetzung des Blutes in verschiedenen Gefässprovinzen. Inaug.-Diss. Breslau 1887. 
ältere mikroskopische und chemische Untersuchungen des Blutes, denen zufolge das Blut der Pfortader reicher an Fett als das anderer Gefässprovinzen sein sollte.

Bor nste in kam zu dem uberraschenden Resultat, dass , me hr Fett in der Carotis als in der Pfortader enthalten" ist, dass sich also die an ihn gestellte Frage, ob Fett ans der Darmschleimhaut direkt in die Pfortaderwurzeln gelangt, durch die chemische Untersuchung des Blutes vor und nach seinem Durchtritt durch die Darmwand nicht entseheiden lasse.

Im Anschluss an diese Versuche vergleicht Bornstein das arterielle und venöse Blut der Unterextremitäten. Er schliesst aus seinen allerdings wenig zahlreichen Beobachtungen, dass auch hier das arterielle Blut reicher an Fett sei, als das venöse, dass also nicht nur in der Darmwand, sondern auch in den anderen Organen dem eintretenden Blute eine nachweisbare Menge Fett entzogen wird.

Eine bald nach der Arbeit von Bornstein veröffentlichte Untersuchnngsreihe von S. Cohnstein and N. Zuntz ${ }^{1}$ ) liess es uns nothwendig erscheinen, die Angaben von Bornstein einer erneuten Prüfung zu unterziehen.

Cohnstein und Zuntz batten nachgewiesen, dass im fliessenden Blute der Gehalt an rothen Blutkörperchen sowohl im Arterien- wie im Venenblute der gleiche ist, dass aber sofort die Zahl der rothen Blutkörperchen im Venenblute zunimmt, wenn auf der venösen Seite der Blutstrom unterbrochen wird. Bleibt nach Unterbindung der Vene die Zufuhr des arteriellen Blutes unbehindert, so wird nur ein relativ kleiner Theil des Blutes durch Collateralen abgeführt, es tritt Blutstauung in dem betreffenden Organ ein, Flïssigkeit filtrirt aus den Capillaren in die Lymphe, die Menge der Blutkörperchen in der Volumenseinheit des Blutes nimmt zu. „Die aus dieser Untersuchung hervorgehende Erkenntniss, dass Behinderung des Venenstromes sehr leicht erhebliche Aenderungen in der Zusammensetzung des Blutes herbeifubrt, dürfte wohl geeignet sein, gewisse ältere Versuche, welche das venöse Blut einzelner Organe erheblich abweichend vom arteriellen fanden, zu discreditiren."

1) Untersuchungen :iber den Flüssigkeits-Austausch zwischen Blut und Geweben unter verschiedenen physiologischen und pathologischen Bedingungen. Dies. Arch. Bd. 42, 303. 
Ueber d. Gehalt ḋes Arterien- u. Venenblutes an Trockensubst. u. Fett. 385

Dieser Satz findet seine Anwendung auch auf die Versuche von Bornstein, welche den Vergleich des Arterien- und Venenblutes der Hinterextremität des Hundes zum Gegenstand haben. Das Blut wurde durch eine endständig in die Vene eingebundene Canüle gewonnen; die Blutèntnahme war also stets mit Stauung verbunden.

Etwas anders liegt die Sache in denjenigen Versuchen, bei denen es sich um einen Vergleich des Arterien- und Pfortaderblutes handelt. Hier wurde die Canüle in eine Milzvene eingeführt, das Blut floss ohne Unterbrechung der Circulation aus der Pfortader ab. Eine Staung ist also nicht ohne weiteres wabrscheinlich.

Nur mit der ersten Gruppe von Versuchen wollen wir uns im Folgenden eingehender beschäftigen. Wir wollen, ebenso wie dies Bornste in beabsichtigte, seben, ob sich der Fettgehalt des Blutes beim Strömen durch die Hinterextremität nachweisbar ändert d. b. abnimmt.

Hierfür ist es nach dem soeben Gesagten in erster Linie nothwendig, dass wir eine Methode befolgen, welche uns gestattet, die zur Analyse erforderlichen Mengen Blutes der Arterie und Vene ohne wesentliche Störung der Circulation zu entziehen.

$\mathrm{Ob}$ eine solche in einem gegebenen Falle eintritt, könnte man nach dem Vorgange von Cobnstein und $\mathrm{Zuntz}$ durch eine vergleichende Zählung der rothen Blutkörperchen des Arterienund Venenblutes zu ermitteln versuchen. Wir thaten dies nicht, sondern benutzten hierzu die Bestimmung des Trockenrickstandes des Blutes.

Dieses Vorgehen wäre im Prinzip mit dem Zählen der rothen Blutkörperchen identisch, wenn wir anf Grund der Beobachtungen von Cohnstein und $\mathrm{Zuntz}$ annehmen könnten, dass nachweisbare Aenderungen im Trockenrtickstand des Venenblutes bei unseren Versuchen im Wesentlichen nur durch eine Aenderung des Verhältnisses zwischen rothen Blutkörperchen und Bluttlissigkeit eintreten können. Da das specifische Gewicht der rothen Blutkörperchen grösser als das des Blutplasmas ist, so müsste der Trockenruickstand des Blutes bei der Staung in dem Maasse zunehmen, als Bluttlüssigkeit aus dem Gefässsystem austritt. Eine Differenz im Trockenrückstand des Arterien- und Venenblutes, bei welcher der Trockenrückstand des letzteren grösser als der des ersteren ist, würde uns umgekehrt ein Beweis für Stanung sein. 
Ehe wir aber aus Veränderungen des Trockenrïckstandes auf Störungen in der Circulation schliessen, müssen wir zuerst die stillschweigend gemachte Voraussetzung, dass bei normaler Circulation kein Untersehied im Trockenrickstand des Arterienund Venenblutes nachweisbar ist, einer experimentellen Prifung unterziehen.

Das arterielle Blut wurde durch die Carotis entnommen. Die Methode, welche sich zur Gewinnung von Blut aus der Vena femoralis als die geeignetste erwies, war folgende.

Bei Hunden wurde die Vene, welche an der inneren Fläche des Oberschenkels, etwa in seiner Mitte, oft schon durch die Hant sichtbar, von innen nach aussen verlänft und sich, nachdem sie von der Tiefe her einige Zweige aufgenommen, in die Vena femoralis einsenkt, sorgfältigst präparirt. Sämmtliche in den Weg kommenden Zweige wurden unterbunden. Die Scheide der Vena femoralis wurde nur soweit eröffnet, dass die Einmündungsstelle unserer Vene sichtbar und zugängig wurde. Nachdem die letztere peripher unterbunden und ein Faden unmittelbar vor ihrer Einmündung in die Vena femoralis umgelegt worden war, wurde sie unter vorsichtigem Anziehen dieses Fadens nahe an der Einmlandungsstelle angeschnitten und eine kurzgeknöpfte, etwa 4 bis 5 mm dicke und $13 \mathrm{~mm}$ lange, an ihrem freien Ende stumpfwinklig abgebogene Canüle eingeführt und mit dem Faden möglichst so eingebunden, dass die Spitze unmittelbar der Einmündungsstelle in die Vene entsprach. Das Blut tropfte nicht, sondern strömte sofort aus der Canüle. Die an der Einmündungsstelle liegenden Klappen bildeten meist kein Hinderniss.

Die zuerst ausfliessenden Mengen wurden sowohl bei der Vene (Femoralis) wie bei der Arterie (Carotis) nicht zur Analyse benutzt.

Das Auffangen des Blutes aus Arterie und Vene geschah selbstverständlich möglichst gleichzeitig.

Wurden mehrere Proben nach einander entnommen, so wurde der Blutstrom während des Wechselns der Gefässe nicht unterbrochen.

Das Blut wurde nicht in Porcellantiegeln getrocknet. Fängt man das Blut in diesen auf, so erhält man stets einen dicken Klumpen, der in seinem Innern leicht etwas Feuchtigkeit einschliessen kann. Wir benutzten deswegen ein System von zwei Schäichen aus diunnem Glase, von denen das eine etwa $5 \mathrm{~cm}$ breit 
Ueber d. Gehalt des Arterien- u. Venenblutes an Trockensubst. u. Fett. 387

und $21 / 2 \mathrm{~cm}$ hoch, das andere $5 \frac{1}{2} \mathrm{~cm}$ breit und $2 \mathrm{~cm}$ hoch war, in beiden waren mit dem Diamant zur Bezeichnung Zahlen eingeritzt. In das erste Schälchen liessen wir soviel Blut einfliessen, dass der Boden eben bedeckt war, dann wurde, um Verdunstung zu vermeiden, schnell das zweite über das erste gestülpt und gewogen. Man erhält so eine dïnne Blutschicht mit grosser Oberfläche, welche leicht und sicher $\mathrm{zu}$ trocknen ist.

Auf diese Weise wurden für den Trockenrückstand des gleichzeitig aufgefangenen Blutes der Arteria carotis und Vena femoralis folgende Zahlen erhalten.

Tabelle I.

\begin{tabular}{|c|c|c|c|c|c|c|c|c|c|}
\hline \multirow[b]{2}{*}{ 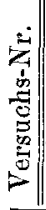 } & \multicolumn{4}{|c|}{ Carotis } & \multicolumn{4}{|c|}{ Vena femoralis } & \multirow{2}{*}{ 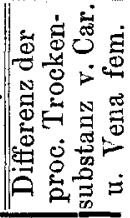 } \\
\hline & $\begin{array}{c}\text { Blut- } \\
\text { menge } \\
g\end{array}$ & $\mid \begin{array}{c}\text { Trocke } \\
\text { absol. } \\
\mathrm{g}\end{array}$ & $\begin{array}{c}\text { asubst. } \\
\text { proc. } \\
\mathrm{g} \\
\end{array}$ & $\begin{array}{l}\text { Differenz d } \\
\text { Trocken- } \\
\text { substanz } \\
\% \\
\end{array}$ & $\begin{array}{c}\text { Blut- } \\
\text { menge } \\
\mathrm{g}\end{array}$ & $\begin{array}{c}\text { Trocke } \\
\text { absol. } \\
\mathrm{g}\end{array}$ & $\begin{array}{c}\text { proc. } \\
\mathrm{g}\end{array}$ & $\begin{array}{c}\text { Differenz d. } \\
\text { Trocken- } \\
\text { substanz } \\
\%\end{array}$ & \\
\hline I. & $\begin{array}{c}5,8082 \\
-\end{array}$ & 1,2918 & 22,76 & - & $\begin{array}{l}6,4220 \\
4,22222\end{array}$ & $\begin{array}{l}1,4431 \\
0,9497\end{array}$ & $\begin{array}{l}22,47 \\
22,49\end{array}$ & 0,02 & \\
\hline II. & $\begin{array}{l}5,4066 \\
4,0370\end{array}$ & 1,0174 & $\begin{array}{l}18,82 \\
18,82\end{array}$ & 0,00 & $\begin{array}{l}3,2955 \\
3,2505\end{array}$ & $\begin{array}{l}0,6210 \\
0,6129\end{array}$ & $\begin{array}{l}18,84 \\
18,85\end{array}$ & 0,01 & - \\
\hline III. & $\begin{array}{l}4,2420 \\
7,7825\end{array}$ & $\begin{array}{l}0,8912 \\
\mathbf{1}, 6297\end{array}$ & $\begin{array}{l}20,99 \\
20,92\end{array}$ & 0,07 & $\begin{array}{l}2,9372 \\
3,8160\end{array}$ & $\begin{array}{l}0,6168 \\
0,7764\end{array}$ & $\begin{array}{l}20,92 \\
20,79\end{array}$ & 0,13 & +0 \\
\hline 18. & 4,3504 & $\begin{array}{l}1,7607 \\
0,96334\end{array}$ & $\begin{array}{l}21,00 \\
21,11\end{array}$ & 0,11 & $\begin{array}{l}4,1574 \\
3,7011\end{array}$ & $\begin{array}{l}0,8746 \\
0,7808\end{array}$ & $\begin{array}{l}21,04 \\
21,10\end{array}$ & 0,06 & $-\mathrm{c}$ \\
\hline
\end{tabular}

In diesen Versuchen wurden sowobl in der Arterie wie in der Vene jedesmal zwei Blutproben nach einander aufgefangen. Die Bestimmung der zweiten Carotisportion in Versuch I verungliuckte.

Die Differenzen im Trockenrückstand zweier nach einander aufgefangener Blutproben von $3-8 \mathrm{~cm}$ derselben Blutart schwanken zwischen 0,00 und $0,13 \%$ und betragen im Mittel etwa $0,06 \%$. Sie geben einen Anhaltspunkt für die Beurtheilung der mit der Trockenbestimmang an sich verbundenen Fehler.

Die Unterschiede im Trockenrückstand von Arterie und Vene liegen in den angeführten 4 Versuchen drei Mal innerhalb dieser Fehler, ein Mal ist der Trockenrückstand der Arterie grösser als der der Vene. 
Die Zahl dieser Versuche ist zu gering, um ein Urtheil über das gegenseitige Verbalten des Trockenruickstandes von Arterie und Vene zu gestatten. Es kommen aber aus den weiter unten anzuführenden Versuchen noch folgende hinzu.

Tabelle II.

\begin{tabular}{|c|c|c|c|}
\hline \multirow[b]{2}{*}{ Versuchs-Nr. } & \multicolumn{2}{|c|}{ Trockenrückstand der } & \multirow[b]{2}{*}{ Unterschied } \\
\hline & $\begin{array}{c}\text { Carotis } \\
\%\end{array}$ & $\underset{\%}{\text { Vena fem. }}$ & \\
\hline XII. & 20,92 & 20,79 & $+0,13$ \\
\hline XIII. & 23,58 & 23,61 & $-0,03$ \\
\hline XIV. & 21,12 & 21,23 & $-0,11$ \\
\hline XV. & 21,17 & 22,22 & $-0,05$ \\
\hline XVI. & 22,88 & 22,87 & $+0,01$ \\
\hline XVIII. & 23,36 & 23,40 & $-0,04$ \\
\hline XVII. & 20,82 & 20,76 & $+0,06$ \\
\hline XIX. & 21,13 & 21,29 & $-0,16$ \\
\hline
\end{tabular}

Hier wurde in Arterie und Vene nur je eine Trockenbestimmung gemacht. Die Differenzen liegen sämmtlich innerhalb der Fehlergrenzen.

Aus diesen und den vorhergehenden Versuchen ergiebt sich, dass sich kein Unterschied im Trockenrückstand des Arterien- und Venenblutes nachweisen lässt, wenn die Blutentnahme ohne Unterbrechung der Circulation erfolgt.

Man kann hierin eine Bestätigung der Ansichten von Flügge sehen. Prinzipiell müssen wir annehmen, dass das Blut bei seinem Durchtritt durch die Gewebe feste Substanzen an dieselbe abgiebt. Nach Flügge können wir trotzdem keine Untersehiede finden, weil unsere Untersuchungsmethoden hierzu nicht ausreichend sind. Die Differenzen, welche in der Zusammensetzung des Arterienund Venenblutes unter dem Einfluss der Gewebe in der Zeiteinheit entstehen, sind infolge der grossen Geschwindigkeit des Blutstromes so klein, dass sie sich dem Nachweis entziehen. Es darf jedoch, wie uns scheint, fur unseren Fall - Bestimmung des Trockenrückstandes - nicht vergessen werden, dass, auch wenn unsere Methode der Trockenrückstandsbestimmung ausreichend genau wäre, die Abgabe von festen Substanzen an die Gewebe unter Umständen durch einen entsprechenden Eintritt von festen Substanzen ins Blut verdeckt werden könnte.

Wie dem auch sei, wir halten uns nur an die Thatsache, dass unter den von uns gewählten Bedingungen ein Unterschied im Trockenrückstand des Arterien- und Venenblutes nicht zu erkennen ist. 
Ueber d. Gehalt des Arterien- u. Venenblutes an Trockensubst. u. Fett. 389

Im Gegensatz hierzu hatte Bornstein den Trockenrickstand der Vene grösser als den der Arterie gefunden (s. d. S. 396 angeführten Versuche). Anf Grund der vorstehend mitgetheilten Erwägungen war es an sich wahrscheinlich, dass dies die Folge der mit der Blutentziehung verbundenen venösen Stauung war.

Wir iiberzeugten uns hiervon direct durch folgende zwei Versuche: In dem einen wurde der Blutstrom in der Vena femoralis während 5 Minuten, in dem anderen während 10 Minuten durch Anlegen einer Klemme unterbrochen. Das hierauf aus der Vena femoralis aufgefangene Blut wurde mit dem gleichzeitig entnommenen Blute der Arteria carotis verglichen.

\begin{tabular}{llc}
\multicolumn{2}{c}{ Trockenrückstand } & Unterschied \\
Arteria carotis & $20,61 \%$ & $0,35 \%$ \\
Vena femoral. & $20,96 \%$ & $0,35 \%$ \\
Arteria carotis & $21,86 \%$ & $0,27 \%$ \\
Vena femoral. & $22,13 \%$ & $0,27 \%$
\end{tabular}

In diesen Fällen ist in der That, wie in den Versuchen von Bornstein, der Trockenriuckstand in der Vene grösser als in der Arterie. Die Unterschiede liegen weit ausserhalb der Fehlergrenzen.

Während also bei ungestörter Circulation kein Unterschied im Trockenrïckstand von Arterie und Vene vorbanden ist, wird bei Staung im Venensystem der Trockenrïckstand in der Vene grösser als in der Arterie.

Unsere Ansicht, dass man aus dem Verhalten des Trockenriickstandes einen Schluss auf das Verhalten der Circulation machen kann, ist demnach thatsächlich begründet.

Bei der Gewinnung des Blutes zum Zweck der Analyse ist noch ein Punkt zu beriicksichtigen.

In den bisherigen Versuchen entnahmen wir zur Bestimmung des Trockenrïckstandes nur kleine Blutproben; für die später anzustellenden Fettbestimmungen bedürfen wir aber erheblich grösserer Blutmengen. Nun ist es eine längst bekannte Thatsache, dass sich das Blut unter dem Einfluss grosser Aderlässe in seiner Zusammensetzung ändert. Man weiss, dass es in diesen Fällen an Farbstoff ärmer, an Wasser reicher wird. Es war deswegen für uns von Werth, zu wissen, wie gross die Abnahme des Trockenrückstandes ist und ob sie, wenn das Blut der Arterie und Vene 
gleichzeitig in grösseren Mengen entzogen wird, in beiden in derselben Weise erfolgt.

$\mathrm{Zu}$ diesem $\mathrm{Z}$ weck wurden unmittelbar vor und nach dem Aderlass Trockenbestimmungen gemacht. In den auf Tabelle III mitgetheilten Versuchen V and VI wurde nur der Carotis, zum Theil in recht ansehnlicher Menge, Blut entzogen. Die Abnahme des Trockenrückstandes ist eine stets sehr deutliche.

Die Versuche XIV bis XIX sind dieselben, in denen das Blut, wie später mitgetheilt werden wird, zur Fettbestimmung benutzt wurde.

Tabelle III.

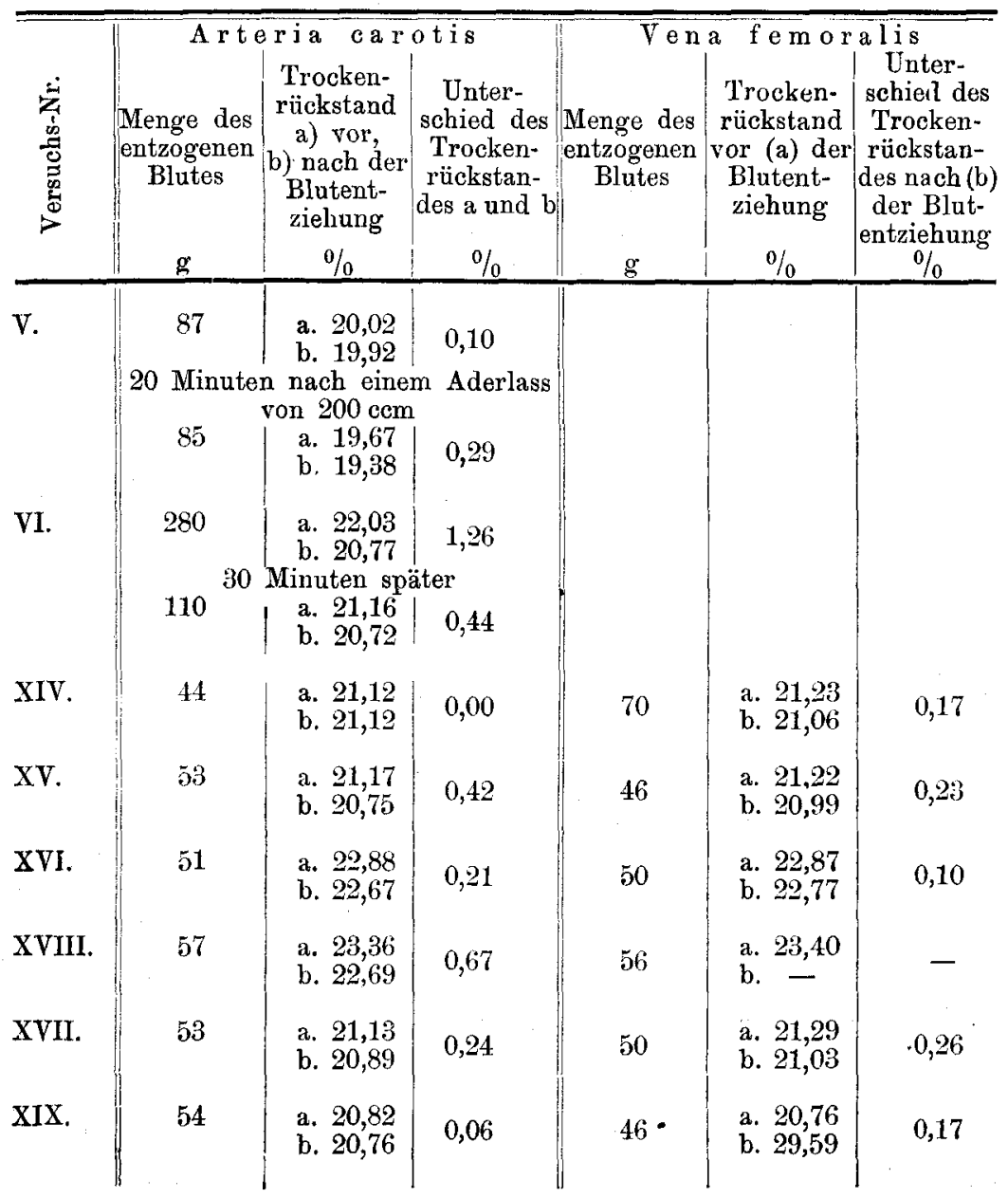


Ueber d. Gehalt des Arterien- u. Venenblutes an Trockensubst. u. Fett. 391

Aus ihnen ergiebt sich, dass schon nach einer Blutentziehung von etwa $50 \mathrm{ccm}$ bei Hunden von 8-10 Kilo fast stets eine Abnahme des Trockenriuckstandes deutlich nachweisbar ist. Dieselbe ist in Beriucksichtigung der Menge des Gesammttrockenriickstandes nur gering und erfolgt in Arterie und Vene in dem gleichen Sinne. Sie ist also, wenn es sich nur um einen Vergleich des Arterien- und Venenblutes in Bezug auf eine in demselben enthaltene Substanz handelt, ohne Bedentung.

Wir können nunmehr zur vergleichenden Fettbestimmung des Arterien- und Venenblutes übergehen.

Die Methode der Fettbestimmung war im Wesentlichen die ron Bornstein beschriebene: In einen $500 \mathrm{ccm} 96 \%$ Alcohol enthaltenden, gewogenen Kolben lässt man unter Umschütteln bis zu einer vorher angebrachten Marke annähernd $50 \mathrm{ccm}$ Blut einfliessen. Die darauf folgende Wägung ergiebt das genaue Gewicht des entnommenen Blutes. Das Blut bleibt bis zum folgenden Tage unter Alcohol stehen. Es wird durch Glaswolle filtrirt. Das auf dem Trichter bleibende Gerinnsel wird mit $200 \mathrm{ccm}$ Aether gewaschen, Alcohol und Aether werden in tiefen Porcellanschalen auf dem Wasserbade verdunstet. Man nimmt den Rückstand mit Aether auf, wobei unter Anderem Salze (Chloride, Phosphate und Sulfate) sowie Zucker ungelöst zurückbleiben und giesst die ätherische Lösung in ein Erlenmeyer'sches Kölbchen. Inzwischen hat man den Blutkuchen auf dem Wasserbade vorsichtig getrocknet und in einer Reibschale zu einem feinen Pulver zerrieben. Man bringt dasselbe behufs vollständiger Extraction in den Soxhlet'schen Apparat, an welchem man das erwähnte, die Hauptmenge des Blutfettes bereits enthaltende Erlenmeyer'sche Kölbchen befestigt. Nach drei- bis vierstiindiger Extraction wurde der Aether verdunstet, der im Kölbchen bleibende Rückstand im Vacuum über Schwefelsäure völlig getrocknet, mit über Natrium destillirtem Aether aufgenommen, in ein kleines, einen Glasstab enthaltendes, gewogenes Becherglas filtrirt, der Aether unter Umrühren mit dem Glasstabe über dem mit Ba u man n'schem Drahtnetz geschïtzten Wasserbade verdunstet und das Zurtickbleibende eine halbe Stunde bei $100^{\circ} \mathrm{C}$. getrocknet. Nach dem Abkühlen über Chlorcalcium werden Becherglas nebst Aetherextract ( ${ }$ Fett") gewogen.

Zur Controlle der Methode wurde der Fettgehalt in 
zwei Blutproben bestimmt, welche der Carotis durch ein Gabelrohr gleichzeitig entzogen worden waren.

$$
\text { Versuch V. }
$$

6,5904 $\mathrm{g}$ Blut geben 1,3206 $\mathrm{g}$ Trockensubstanz: 20,0231\%

a) $45,7 \mathrm{~g}$ Blut enthalten $0,4179 \mathrm{~g}$ Fett: $0,9144 \%$ $41,7 \mathrm{~g} \quad, \quad, \quad 0,4011 \mathrm{~g} \quad, \quad 0,9619 \%$ 6,2602 g Blut geben 1,2471 g Trockensubstanz: 19,9211\% ca. $200 \mathrm{ccm}$ Blut werden abgelassen, nach $20 \mathrm{Min}$.

b) 4,2215 g Blut geben $0,8305 \mathrm{~g}$ Trockensubstanz: $19,6741 \%$ 42,2 $\mathrm{g}$ Blut enthalten 0,4073 $\mathrm{g}$ Fett: $0,9652 \%$ $43,4 \mathrm{~g} \quad \# \quad \# \quad 0,4017 \mathrm{~g} \quad, \quad 0,9256 \%$ 5,0329 $\mathrm{g}$ Blut geben 0,9742 $\mathrm{g}$ Trockensubstanz: 19,3762\%

Versuch VI.

a) $3,4147 \mathrm{~g}$ Blut geben 0,7523 $\mathrm{g}$ Trockeńsubstanz: 22,0312\% $57,0 \mathrm{~g}$ Blut enthalten $0,4259 \mathrm{~g}$ Fett: $0,7470 \%$ $47,0 \mathrm{~g}, \quad, \quad 0,3490 \mathrm{~g} \quad, \quad 0,7430 \%$ ca. $200 \mathrm{ccm}$ Blut werden abgelassen $3,6559 \mathrm{~g}$ Blut geben 0,7594 g Trockensubstanz: 20,7719\%

30 Minuten später

b) $3,5580 \mathrm{~g}$ Blut geben 0,7528 $\mathrm{g}$ Trockensubstanz: $21,1579 \%$ $55,3 \mathrm{~g}$ Blut enthalten $\left[0,3908{ }^{1}\right)$ Fett: $\left.0,7067 \%\right]$ $54,8 \mathrm{~g} \quad " \quad 0,4007 \mathrm{~g} \# \quad 0,7191 \%$ 3,3901 g Blut geben 0,6984 $\mathrm{g}$ Trockensubstanz: 20,7191\%

Die Werthe für den Fettgehalt zeigt die folgende Tabelle. Tabelle IV.

\begin{tabular}{c|c|c|c}
\hline \hline $\begin{array}{c}\text { Versuchs- } \\
\text { Nr. }\end{array}$ & $\begin{array}{c}\text { Fettgehalt in } \\
100 \text { g Carotis- } \\
\text { blut }\end{array}$ & $\begin{array}{c}\text { Unterschied } \\
\text { zweier } \\
\text { Bestimmungen }\end{array}$ & Bemerkung \\
\hline V a & $\begin{array}{c}0,914 \\
0,962\end{array}$ & 0,048 & $\begin{array}{c}\text { a vor, b nach einem Aderlass von } \\
\text { etwa 200 ccm. }\end{array}$ \\
b & $\begin{array}{c}0,965 \\
0,926\end{array}$ & 0,039 & \\
VI a & $\begin{array}{c}0,747 \\
0,743\end{array}$ & 0,004 & \\
b & $\begin{array}{c}0,707] \\
0,719\end{array}$ & {$[0,012]$} &
\end{tabular}

In der folgenden Versuchsreibe wurden die beiden Blutportionen, deren Fettgehalt mit einander verglichen werden sollte, unmittelbar nacheinander ans der Carntis aufgefangen.

1) Geringer Verlust bei der Bestimmung. 
Ueber d. Gehalt des Arterien- u. Venenblutes an Trockensubst. u. Fett 393

Tabelle V.

\begin{tabular}{|c|c|c|c|c|}
\hline $\begin{array}{c}\text { Versuchs- } \\
\text { Nr. }\end{array}$ & $\begin{array}{c}\text { Blut aus } \\
\text { Carotis } \\
g\end{array}$ & $\begin{array}{r}\text { Fe } \\
\text { absolute Menge } \\
\mathbf{g}\end{array}$ & $\begin{array}{l}\mathrm{t} t \\
\text { Procentgehalt } \\
\quad \mathrm{g}\end{array}$ & $\begin{array}{l}\text { Unterschied zweier } \\
\text { Bestimmungen des } \\
\text { procentischen Fett- } \\
\text { gehaltes }\end{array}$ \\
\hline VII. & $\begin{array}{l}51,5 \\
52,2\end{array}$ & $\begin{array}{l}0,3868 \\
0,3915\end{array}$ & $\begin{array}{l}0,751 \\
0,750\end{array}$ & 0,001 \\
\hline VIII. & $\begin{array}{l}57,0 \\
53,9\end{array}$ & $\begin{array}{l}0,4368 \\
0,3868\end{array}$ & $\begin{array}{l}0,706 \\
0,716\end{array}$ & 0,010 \\
\hline IX. & $\begin{array}{l}52,2 \\
51,1\end{array}$ & $\begin{array}{l}0,4898 \\
0,4942\end{array}$ & $\begin{array}{l}0,938 \\
0,967\end{array}$ & 0,029 \\
\hline $\mathrm{x}$. & $\begin{array}{l}39,4 \\
46,8\end{array}$ & $\begin{array}{l}0,3133 \\
0,3552\end{array}$ & $\begin{array}{l}0,794 \\
0,759\end{array}$ & 0,035 \\
\hline XI. & $\begin{array}{l}40,8 \\
45,9\end{array}$ & $\begin{array}{l}0,3013 \\
0,3451\end{array}$ & $\begin{array}{l}0,725 \\
0,752\end{array}$ & 0,027 \\
\hline
\end{tabular}

Wir sehen aus diesen 8 Doppelbestimmungen, dass der procentische Fettgehalt zweier durch ein Gabelrohr gleichzeitig, oder durch eine einfache Canüle unmittelbar nacheinander aufgefangene Portionen desselben Butes einen Unterschied von 0,001 bis $0,048 \%$ zeigen kann.

Aus dem bisher Mitgetheilten folgt, dass es möglich ist, ohne wesentliche Störung der Circulation dem Arterien- und Venenblute die zur Analyse erforderlichen Mengen zu entziehen und dass wir iiber eine Methode verfügen, welche es uns gestattet, den Fettgehalt des Blutes mit grosser Genauigkeit zu bestimmen.

Erst jetzt sind wir im Stande, mit einer gewissen Sicherheit an die Frage, welche bereits Bornstein zu beantworten suchte, heranzutreten: Aendert sich der Fettgehalt des Blutes beim Strömen durch die Hinterextremität des Hundes?

$\mathrm{Zu}$ den diesbezüglichen Versuchen dienten 8-12 Kilo schwere Hunde, welche nach dreitägigem Hungern am Morgen des Versucbstages in den 5 ersten Versuchen $500 \mathrm{~g}$ Fleisch und $500 \mathrm{~g}$ Schweinefett, in den letzten dreien nur $250 \mathrm{~g}$ Butter erhalten hatten. In der 3 . bis 6 . Verdauungsstunde wurden sie morphinisirt. Das Blut wurde in der vorstehend beschriebenen Weise gleichzeitig der Arteria carotis und Vena femoralis entnommen und verarbeitet. Vor und nach jeder grösseren Blutentziehung wurde in kleineren Proben der Trockenriickstand bestimmt. 
Versuch XII.

Arterie 7,7825 g Blut $-1,6281 \mathrm{~g}$ Trockensubstanz $=20,9200 \%$ $47,1 \mathrm{~g}$ Blut $-0,3648 \mathrm{~g}$ Fett $=0,7440 \%$

Fett im Trockenrückstand $3,55 \%$

Vene $\quad 3,8160 \mathrm{~g}$ Blut $-0,7933 \mathrm{~g}$ Trockensubstanz $=20,7861 \%$

$54,5 \mathrm{~g}$ Blut $-0,3874 \mathrm{~g}$ Fett $=0,7100 \%$

Fett berechnet auf Trockenriickstand $3,41 \%$

Versuch XIII.

Art. $7,1968 \mathrm{~g}$ Blut $-1,6972 \mathrm{~g}$ Trockensubstanz $=23,5828 \%$ $58,8 \mathrm{~g}$ Blut $-0,4843 \mathrm{~g}$ Fett $=0,8246 \%$ Fett im Trockenrückstand $3,49 \%$

Ven. $3,7621 \mathrm{~g}$ Blut $-0,8912 \mathrm{~g}$ Trockensubstanz $=23,6091 \%$ $68,7 \mathrm{~g}$ Blut $-0,4977 \mathrm{~g}$ Fett $=0,7259 \%$ Fett im Trockenrückstand $3,08 \%$

Versuch XIV.

Art. $\quad 6,2358 \mathrm{~g}$ Blut $-1,3170 \mathrm{~g}$ 'Trockensubstan $\angle=21,1190 \%$ $44,5 \mathrm{~g}$ Blut $\ldots . .0,3009 \mathrm{~g}$ Fett $=0,6761 \%$ Fett im Trockenrückstand $3,20 \%$

$4,3270 \mathrm{~g}$ Blut $-0,9138 \mathrm{~g}$ Trockensubstanz $=21,1190 \%$

Ven. $4,0943 \mathrm{~g}$ Blut $-0,8692 \mathrm{~g}$ Trockensubstan $z=21,2290 \%$ $70,3 \mathrm{~g}$ Blut $-0,4782 \mathrm{~g}$ Fett $=0,6802 \%$ Fett im Trockenrückstand 3,21\% $4,0017 \mathrm{~g}$ Blut $-0,8428 \mathrm{~g}$ Trockensubstanz $=21,0620 \%$

Versuch XV.

Art. 5,5646 g Blut $-1,1780 \mathrm{~g}$ Trockensubstan $=21,1695 \%$ $53,3 \mathrm{~g}$ Blut $-0,4892 \mathrm{~g}$ Fett $=0,9183 \%$ Fett im Trockenrückstand $4,39 \%$

$3,6546 \mathrm{~g}$ Blut $-0,7584 \mathrm{~g}$ 'Trockensubstanz $=20,7518 \%$

Ven. $5,1100 \mathrm{~g}$ Blut $-1,0844 \mathrm{~g}$ Trockensubstanz $=21,2231 \%$ $46,5 \mathrm{~g}$ Blut $-0,4142 \mathrm{~g}$ Fett $=0,8906 \%$ Fett im Trockenrückstand $4,22 \%$

$3,5335 \mathrm{~g}$ Blut $-0,7460 \mathrm{~g}$ Trockensubstanz $=20,9910 \%$

Versuch XVI.

Art. $6,0663 \mathrm{~g}$ Blut $-1,3878 \mathrm{~g}$ Trockenrückstand $=22,8772 \%$ $51,1 \mathrm{~g}$ Blut $-0,3542 \mathrm{~g}$ Fett $=0,6932 \%$ Fett im 'Trockenrückstand $3,04 \%$

$3,4024 \mathrm{~g}$ Blut $-0,7715 \mathrm{~g}$ Trockenrückstand $=22,6751 \%$

Ven. 5,1476 g Blut $-1,1773 \mathrm{~g}$ Trockenrückstand $=22,8708 \%$ $50,2 \mathrm{~g}$ Blut $-0,3557 \mathrm{~g}$ Fett $=0,7016 \%$

Fett im Trockenrückstand $3,07 \%$ $3,5525 \mathrm{~g}$ Blut $-0,8091 \mathrm{~g}$ Trockenrückstand $=22,7755 \%$ 
Ueber d. Gehalt des Arterien- u. Venenblutes an Trockensubst. u. Fett. 395

Versuch XVII.

Art. $4,9380 \mathrm{~g}$ Blut - $1,1434 \mathrm{~g}$ Trockenrückstand $=21,13 \%$

$53,3 \mathrm{~g}$ Blut $-0,3692 \mathrm{~g}$ Fett $=0,693 \%$

$4,8278 \mathrm{~g}$ Blut $-1,0088 \mathrm{~g}$ Trockenrückstand $=20,89 \%$

Ven. $2,7892 \mathrm{~g}$ Blut $-0,6030 \mathrm{~g}$ Trockenrückstand $=21,29 \%$

$50,3 \mathrm{~g}$ Blut $-0,3532 \mathrm{~g}$ Fett $=0,702 \%$

$7,6382 \mathrm{~g}$ Blut $-1,6062 \mathrm{~g}$ Trockenrückstand $=21,03 \%$

Versuch XVIII.

Art. $2,8866 \mathrm{~g}$ Blut $-0,6744 \mathrm{~g}$ Trockenrückstand $=23,36 \%$

$57,5 \mathrm{~g}$ Blut $-0,3848 \mathrm{~g}$ Fett $=0,668 \%$

$3,4412 \mathrm{~g}$ Blut $-0,7810 \mathrm{~g}$ Trockenrückstand $=22,69 \%$

Ven. $5,8234 \mathrm{~g}$ Blut $-1,3626 \mathrm{~g}$ Trockenrückstand $=23,40 \%$

$55,9 \mathrm{~g}$ Blut $-0,3760 \mathrm{~g}$ Fett $0,671 \%$

Beim Auffangen des Blutes zur zweiten Trockenbestimmung tritt Gerinnung ein.

Versuch XIX.

Art. $2,9752 \mathrm{~g}$ Blut $-0,6194 \mathrm{~g}$ Trockenrückstand $=20,82 \%$

$53,9 \mathrm{~g}$ Blut $-0,4636 \mathrm{~g}$ Fett $=0,860 \%$

$2,5624 \mathrm{~g}$ Blut $-0,5320 \mathrm{~g}$ Trockenrïckstand $=20,76 \%$

Ven. $4,9834 \mathrm{~g}$ Blut $-1,0346 \mathrm{~g}$ Trockenrückstand $=20,76 \%$

$46,3 \mathrm{~g}$ Blut $-0,3976 \mathrm{~g}$ Fett $=0,859 \%$

$5,2208 \mathrm{~g}$ Blut $-1,0754 \mathrm{~g}$ Trockenrückstand $=20,59 \%$

In der folgenden Tabelle sind die Zahlen für das Fett zusammengestellt.

Tabelle VI.

\begin{tabular}{l|c|c|c}
\hline Versuchs-Nr. & $\begin{array}{c}\text { Fett in 100 g } \\
\text { Gesammtblut } \\
\text { Art. carotis } \\
\%\end{array}$ & $\begin{array}{c}\text { Fett in 100 g } \\
\text { Gesammtblut } \\
\text { Vena femor. } \\
\% \%\end{array}$ & Unterschied \\
\hline XII. & 0,744 & 0,710 & $+0,034$ \\
XIII. & 0,825 & 0,726 & $+0,099$ \\
XIV. & 0,676 & 0,680 & $-0,004$ \\
XV. & 0,918 & 0,890 & $+0,027$ \\
XVI. & 0,693 & 0,702 & $-0,009$ \\
XVII. & 0,693 & 0,702 & $-0,009$ \\
XVIII. & 0,668 & 0,671 & $-0,003$ \\
XIX. & 0,860 & 0,859 & $-0,001$
\end{tabular}

Unter diesen 8 Versuchen findet sich nur einer (Versuch XIII), bei welchem der Unterschied des procentischen Fettgebaltes ausserhalb der Fehlergrenzen liegt. Man wird denselben wohl als durch einen Versuchsfehler bedingt betrachten können.

Besonders in den letzten Versuchen sind die Unterschiede des Fettgehalts in Arterie und Vene noch kleiner als in den vor- 
stehend angeführten Controllbestimmungen. Ob dies nur die Folge der mit der Zeit erlangten grösseren Sicherheit in der Beherrschung: der Methode ist, oder ob der Zufall seine Hand im Spiele hatte, wagen wir nicht $z u$ entscheiden. Jedenfalls ist so viel sicher, dass bei ungestörter Circulation ein Unterschied im Fettgehalt des Blutes der Arteria carotis und Vena femoralis nicht nachzuweisen ist.

Vergleichen wir hiermit die Versuche von Bornstein.

Tabelle VII.

\begin{tabular}{|c|c|c|c|c|c|c|c|}
\hline 安 & \multicolumn{2}{|c|}{ Trockenrïckstand } & \multicolumn{2}{|c|}{$\begin{array}{l}\text { Fett in } 100 \mathrm{~g} \\
\text { Gesammtblut }\end{array}$} & \multicolumn{2}{|c|}{$\begin{array}{l}\text { Fett in } 100 \mathrm{~g} \\
\text { Trockensubstanz }\end{array}$} & \multirow[t]{2}{*}{$\begin{array}{l}\text { Bemer- } \\
\text { kungen }\end{array}$} \\
\hline$\stackrel{\Phi}{2}$ & Carotis & Vena cava & Carotis & Vena cava & Carotis & Vena cava & \\
\hline 12 & 22,47 & 24,87 & 0,562 & 0,577 & 2,50 & 2,32 & Born- \\
\hline 13 & 20,72 & 21,84 & 0,938 & 0,884 & 4,53 & 4,05 & stein's \\
\hline 14 & 16,94 & 20,86 & 1,096 & 1,045 & 6,47 & 5,01 & Tab. III. \\
\hline & Art. fem. & Vena fem. & Art. fem. & Vena fem. & Art. fem. & Vena fem. & \\
\hline 15 & 22,96 & 23,79 & 0,833 & 0,647 & 3,63 & 2,79 & () Born- \\
\hline 16 & 23,22 & 22,65 & $0,7.19$ & 0,666 & 3,10 & 2, & $\begin{array}{l}7 \text { stein's } \\
\text { Tab. IV }\end{array}$ \\
\hline
\end{tabular}

Bei einer Betrachtung derjenigen Zahlen, welche den Fettgehalt des Blutes anf Trockenrückstand berechnet angeben, erscheint der Schluss Bornstein's nicht ungerechtfertigt, dass im Arterienblut mehr Fett enthalten ist, als in dem der Vene. Sehen wir uns die Werthe für den Procentgehalt des Gesammtblutes an Fett an, so finden wir futr den Unterschied im procentischen Fettgehalt der Arterie und Vene folgende Zahlen.

Fettgehalt der Carotis minus Vena cava inf.

$$
\begin{array}{r}
0,015 \\
+0,054 \\
+0,051
\end{array}
$$

Fettgehalt der Arteria femoralis minus vena femoralis.

$$
\begin{array}{r}
+0,186 \\
+0,053
\end{array}
$$

In einem Falle liegt die Differenz innerhalb der Fehlergrenzen, in dreien denselben sehr nahe, nur in einem mit Sicherheit ausserhalb der Fehlergrenzen.

Es ist die Frage, ob man diese Versuche in Anbetracht ibrer geringen Zahl und der kleinen gefundenen Differenzen ïberhaupt berticksichtigen soll. Wir wollen unsere Ansicht in folgender Weise ausdrücken: Wir würden es für sehr wahrscheinlich halten, 
Ueber d. Gehalt des Arterien- u. Venenblutes an Trockensubst. u. Fett. 397

dass man, wenn die Versuche unter denselben Bedingungen, unter denen sie Bornstein anstellte, wiederholt wiirden, dasselbe Resultat wie Bornstein erhielte. Der Fettgehalt der Arterie würde grösser zu sein scheinen als der der Vene; und zwar aus folgendem Grunde: Die Blutentnahme erfolgte in Bornstein's Versuchen, wie wir gesehen haben, unter Stauung im Venensystem (Einführung eines Katheters durch das Herz bis in die Vena cava inferior, Einbinden einer Canüle in das periphere Ende der Vena femoralis). Dieselbe musste nach Cohnstein und $\mathrm{Zuntz}$ eine Anhäufung von rothen Blutkörperehen in der Vene zur Folge haben. Da nun das Fett zum allergrössten Theil im Blutplasma enthalten ist, so muss bei der Stauung nothwendiger Weise der procentische Fettgehalt des Venenblutes herabgedriickt werden, der Fettgehalt des Arterienblutes vergleichsweise höher erscheinen. Demnach wären die Beobachtungen Bornstein's, insofern sie sich auf das Stauungsblut beziehen, zwar richtig, der aus ihnen für das fliessende Blut gezogene Schluss dagegen nicht zutreffend.

Auf die Versuche von Bornstein, welche den Vergleich des Arterien- und Pfortaderblutes betreffen, kommen wir vielleicht bei späterer Gelegenheit zurück.

Das Resultat der vorstehend mitgetheilten Versuche ist zunächst nur ein negatives, insofern es nicht gelang, am narkotisirten Thiere, bei ungestörter Circulation, einen Unterschied zwischen arteriellem und venösem Blut der Extremitäten in Bezug auf seinen Gebalt an Trockensubstanz und Fett aufzufinden. Es ist dies besonders bemerkenswerth für das Fett, welches alle anderen Extractivstoffe bei weitem iubertrifft.

Mancher wird hierin, wie wir dies bereits oben andeuteten, nur eine Bestätigung der Ansicht von Flïgg e finden, dass es aussichtslos ist, durch einen Vergleich des Arterien- und Venenblutes Aufschluss uber die Funktion eines Organs zu erhalten.

Wäre dieselbe aber in ihrer ganz allgemeinen Fassung richtig, so missten Angaben wie die von Chaveau und Kauffmann, welche eine Abnahme von Zucker im Blut unter dem Einfluss der Thätigkeit fanden, einer Nachprüfung unterzogen werden.

Im anderen Falle wilrden die von uns angestellten Versuche die Basis fur weitere Untersuchungen uber Beziehungen zwischen Function der Organe, speciell der Muskeln, und Fettgebalt des Blutes geben können. 